\title{
Pleuropneumonia with chest wall abscess due to salmonella enteric in immunocompetent patient: a case report
}

\author{
Youssef Sleiman'*, Mazen Zaylaa ${ }^{1}$, Ahmad El Fay ${ }^{2}$, Jihad Youssef ${ }^{2}$, Kamar Eid $^{2}$ and Chadi Hamdach ${ }^{3}$ \\ ${ }^{1}$ Department of Surgery, Nini Hospital, Tripoli, Lebanon. \\ ${ }^{2}$ Department of internal medicine, Nini Hospital, Tripoli, Lebanon. \\ ${ }^{3}$ Department of Microbiology, Kadisha Medical Laboratories, Tripoli, Lebanon.
}

*Corresponding Author: Youssef Sleiman, Department of Surgery, Nini Hospital, Tripoli, Lebanon,

Email: youssef.ab.sleiman@gmail.com.

Received date: July 23, 2020; Accepted date: July 31, 2020; Published date: September 08, 2020

Citation: Youssef Sleiman, Mazen Zaylaa, Ahmad El Fay, Jihad Youssef, Kamar Eid and Chadi Hamdach, (2020) Pleuropneumonia with chest wall abscess due to salmonella enteric in immunocompetent patient: a case report; J Clinical Medical Reviews and Reports. 2(5); DOI: 10.31579//2690-8794//025

Copyright: ( 2020 , Youssef Sleiman, This is an open access article distributed under the Creative Commons Attribution License, which permits unrestricted use, distribution, and reproduction in any medium, provided the original work is properly cited.

\begin{abstract}
Introduction: Pleuropulmonary with bony and cutaneous involvement of salmonella infection is a very rare condition and no case was ever reported in Lebanon.

Case presentation: We report a case of 39-year-old immunocompetent man, with no past medical history, presented with a right bulging chest wall mass. The chest radiographs revealed pleural effusion and presence of a hypodense central necrosis at right lower lung field. Salmonella was identified from the cultures of fine needle aspiration (FNA) form the mass. After treating salmonella with adequate antibiotics, the patient was improved.

Discussion: salmonella infection is a common bacterial disease that affects the intestinal tract. Infection may spread beyond the intestine causing many complications like in our reported case.

Conclusion: we should suspect salmonellosis in any patient presenting with gastrointestinal symptoms followed by pleuropneumonia with chest wall infection.
\end{abstract}

Keywords: Pleuropneumonia; salmonella enteric; immunocompetent

\section{Introduction}

Salmonella belongs to the family Enterobacteriaceae that is motile Gram-negative, non-spore forming facultative anaerobic bacilli [1]. Salmonella enterica mostly causes enterocolitis and typhoid fever [2]. Extra-intestinal invasion such as bacteremia or localized infections including osteomyelitis, nephritis, cholecystitis and meningitis had been described [3]. However, Salmonella Infection outside of the gastrointestinal tract remains uncommon. Pulmonary and cutaneous infection due to Salmonella enteric is an extremely rare emerging infection occurring in the form of bronchopneumonia, with subcutaneous abscess. Worldwide, few cases were reported, the majority seen in immunocompromised patient (HIV positive), or associated with an underlying disease such as lung cancer, diabetes mellitus, pulmonary disease and osteomyelitis in sickle cell anemia [4]. We report a case of pulmonary salmonella infection with cutaneous involvement in a 39 years old immunocompetent patient, and a brief literature review about this topic.

\section{Case presentation}

39 year old male smoker, previously healthy, presented to our outpatient department with a painful subcutaneous nodule over his anterior chest wall right to the xyphoid process, pain was radiating to the right shoulder, he complained of dyspnea on minimal exertion and non-productive cough. The nodule appeared 2 months ago but was asymptomatic; he started to have nocturnal spiking fever that started 2 weeks prior to presentation. In review of system, the patient reported a watery, non-bloody diarrhea since 3 weeks.

Upon presentation, blood pressure was $100 / 60 \mathrm{mmHg}$, pulse rate $84 / \mathrm{min}$, respiration rate $25 / \mathrm{mins}$, temperature $37{ }^{\circ} \mathrm{C}$, and oxygen saturation $99 \%$ on room air.

Physical exam revealed erythematous warmth subcutaneous mass on his chest wall, decreased breath sounds on auscultation and dullness to percussion over the right lung base while these were negative on the left lung. The mass was hard, firm non erythematous but tender upon touching. The laboratory findings were as follows: White Blood Cell count of 14, 2 $\times 10^{9} / \mathrm{L}$. Hemoglobin of $12.5 \mathrm{~g} / \mathrm{dL}$. C-reactive protein of $79 \mathrm{mg} / \mathrm{l}$. renal function test was found to be normal with serum Creatinine of $0,76 \mathrm{mg} / \mathrm{dl}$ and blood urea nitrogen. Liver function tests were also normal. No other pathological findings were indicated from the biochemical testing. 
Postero-anterior Chest radiography (Fig.1) showed blunting of the costophrenic angle on the right side, which indicates right pleural effusion. Computed tomography CT scan (Fig.2) showed right moderate pleural effusion associated with triangular opacity at the base of the lung with air Bronchogram.

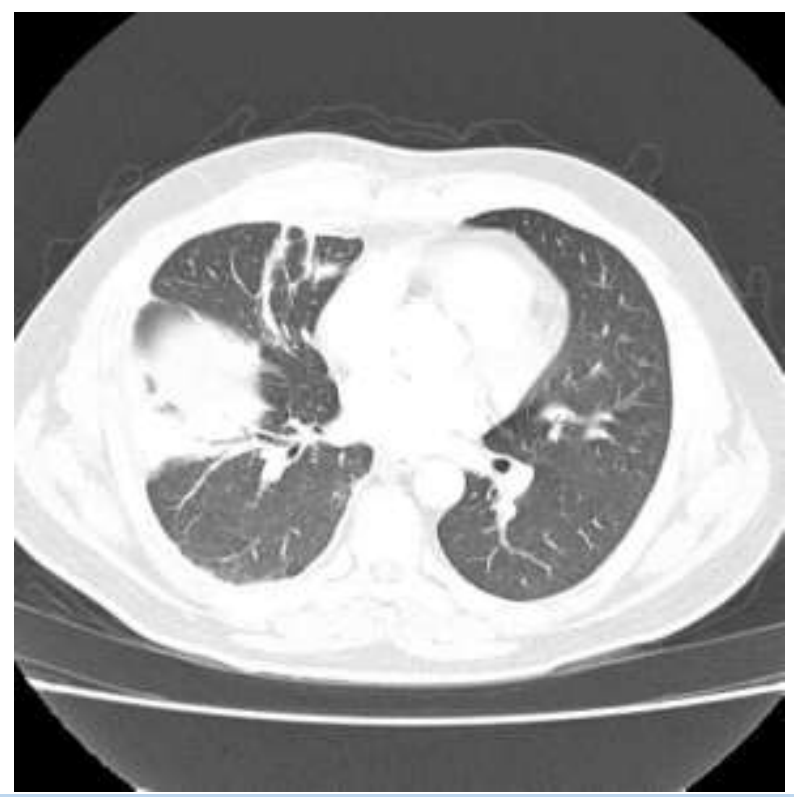

Fig.1: blunting of the costophrenic angle on the right side, which indicates right pleural effusion

Fig.2: right moderate pleural effusion associated with triangular opacity at the base of the lung with air Bronchogram.

A diagnostic FNA from the subcutaneous mass was performed, and sent to laboratory for biochemical analysis, Gram stain, cultures, and antimicrobial profile. Biochemical analysis revealed a turbid exudative fluid with White blood cell count of 12200/mm3 (85\% Neutrophils, $10 \%$ Lymphocytes, $3 \%$ Eosinophils and $2 \%$ Monocytes) and Red blood cells count of $50000 / \mathrm{mm} 3$.

Acid fast bacilli (AFB) staining for mycobacteria was also performed on the fluid sample and the result was negativeCultures of the fluid sample collected from the abscess yielded a Gram-negative aerobic rod identified as Salmonella enteric. Salmonella isolate was identified to the genus level by the standard conventional biochemical methods through glucose and lactose fermentation, urease reaction, lysine decarboxylase, indole test, $\mathrm{H}_{2} \mathrm{~S}$ production and by API 20E (bioMerieux, Inc., Hazelwood, MO).

Confirmation of Salmonella at species level was carried out by MaldiTOF MS technique (bioMérieux, France).

Antimicrobial susceptibility testing was performed using the standardized Kirby-Bauer disk diffusion technique, in accordance with the European Committee on Antimicrobial Susceptibility Testing (EUCAST) for Antimicrobial Susceptibility Testing guidelines. Susceptibility testing showed sensitivity to Amoxicillin, Piperacillin, Ticarcillin, Cephalosporin, Trimethoprim-sulfamethoxazole, Aztreonam, Meropenem, Imipenem, Gentamicin, Amikacin, Tobramycin and Colistin. According to these results, the patient was treated with oral 
Bactrim (Sulfamethoxazole / trimethoprim) for a period of 6 months. Follow-up blood cultures became negative after 7 days of treatment. No additional cultures of the pleural fluid after drainage were performed.

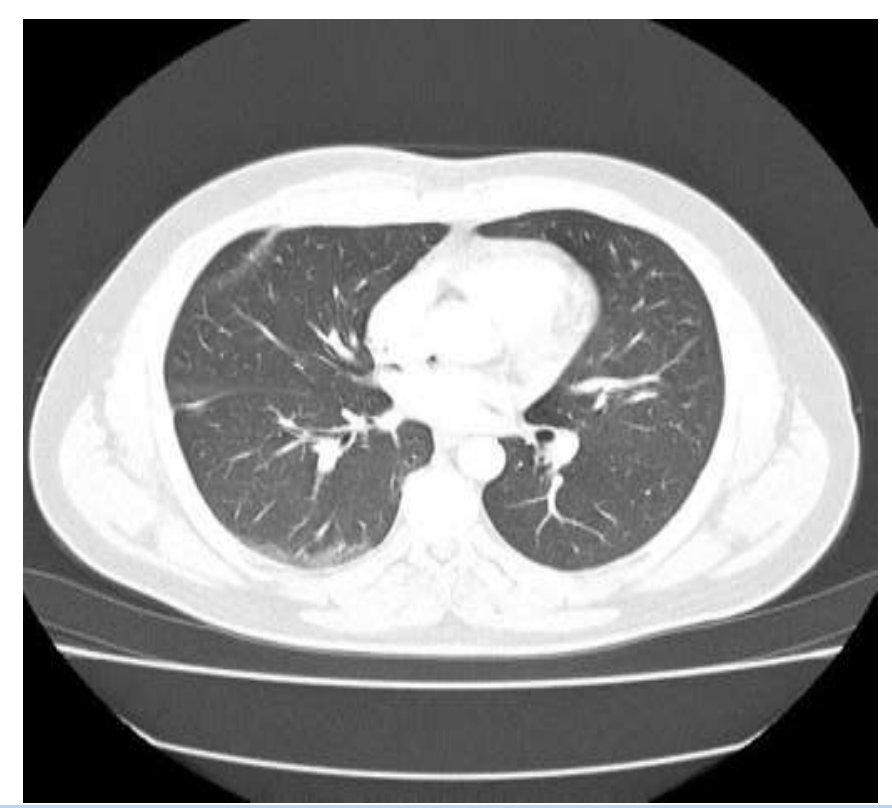

Fig.3: this $\mathrm{x}$ ray confirms the disappearance of the right pleural effusion.

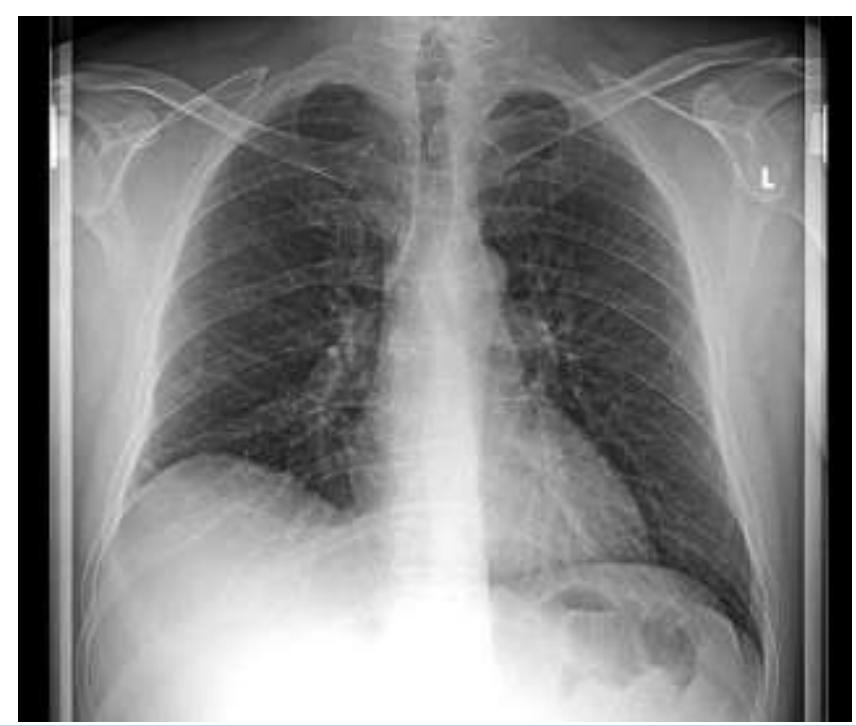

Fig.4: Disappearance of right basal alveolar opacity a long with the right pleural effusion. Persistance of a thin bosa-lateral atelectasis band.

\section{Discussion}

Salmonella infection (salmonellosis) is a common bacterial disease that affects the intestinal tract. Salmonella typically live in animal and human intestines and are shed through feces.

Life threatening complications, like in our reported case, may develop if the infection spreads beyond the intestines, especially in infants and young children, older adults, transplant recipients, pregnant women, and people with weakened immune systems.

In recent years, several cases of salmonella infection with extra intestinal manifestations have been reported, such as urinary tract infection, meningitis, osteomyelitis, pneumonia and soft tissue infection.
Pneumonia, with or without pleural empyema, and soft tissue abscess represent uncommon complication of NTS infection, accounting for 10 $\%$ [5] , and from 3,7 \% [6], to 7,3\%[7], respectively, of extra-intestinal focal infections. To the best of our knowledge, chest wall abscesses

caused by NTS have been reported only in 6 patients between 1990 and 2011 [8]. This is the first reported case in Lebanon.

It is estimated that perhaps $1 \%$ of enteric infections with NTS result in bacteremia [9]. The burden varies georgraphically.in a systemic review that extrapolated the global incidence of invasive NTS infections based on 10 studies, there were an estimated 49 cases per 100000 people worldwide in 2010 [10] . Incidence of 227 cases per 100000 people. $65 \%$ of cases worldwide are in children younger than 5 years [10]. 
Typhoidal salmonella co-opt the macrophages cellular machinery for their own reproduction as they are carried through the mesenteric lymph nodes to the thoracic duct and the lymphatic and then through to the reticuloendothelial tissues of the liver, spleen, bone marrow, and lymph nodes. Once there, they pause and continue to multiply until some critical density is reached. Afterward, the bacteria induce macrophage apoptosis, breaking out into the bloodstream to invade the rest of the body. That is how they can reach the lungs and the pleura and by contiguity, they can invade the soft tissue manifesting as abscess.

Extra intestinal NTS infections generally require surgical drainage or debridement and prolonged antimicrobial therapy fluoroquinolones are a reasonable empiric antibiotic of choice. Third generation cephalosporin, trimethoprim-sulfamethoxazole are reasonable alternative.

\section{Conclusion}

Bacteremia and other forms of extra intestinal salmonella infection as pleuropulmonary and soft tissue involvements are quite uncommon, but should be suspected in any patient presenting with gastrointestinal symptoms followed by pneumonia with chest wall infection.

\section{Reference}

1. Bell, R.L., Jarvis, K.G., Ottesen, A.R., McFarland, M.A., Brown, E.W., (2016). Recent and emerging innovations in Salmonella detection: a food and environmental perspective. Microb Biotechnol 9, 279-292.

2. Giannella, R.A., (1996). Salmonella, in: Baron, S. (Ed.), Medical Microbiology. University of Texas Medical Branch at Galveston, Galveston (TX).

3. Adhikary, R., Joshi, S., Ramakrishnan, M., (2013). Salmonella typhimurium meningitis in infancy. Indian J Crit Care Med 17, 392-393.

4. Bastin, M.L.T., Neville, N.R., Parsons, R.E., Flannery, A.H., Tennant, S.J., Johnson, C.A., (2016). An unusual case of Salmonella Enteritidis causing pneumonia, septic shock and multiple organ failure in an immunocompetent patient. IDCases 6,85 .

5. Chen, P.-L., Chang, C.-M., Wu, C.-J., Ko, N.-Y., Lee, N.-Y., Lee, H.-C., Shih, H.-I., Lee, C.-C., Wang, R.-R., Ko, W.-C., (2007). Extraintestinal focal infections in adults with nontyphoid Salmonella bacteraemia: predisposing factors and clinical outcome. J. Intern. Med. 261, 91-100.

6. Fisker, N., Vinding, K., Mølbak, K., Hornstrup, M.K., 2003. Clinical review of nontyphoid Salmonella infections from 1991 to 1999 in a Danish county. Clin. Infect. Dis. 37, e47-52.

7. Dhanoa, A., Fatt, Q.K., (2009). Non-typhoidal Salmonella bacteraemia: epidemiology, clinical characteristics and its' association with severe immunosuppression. Ann. Clin. Microbiol. Antimicrob. 8, 15.

8. Fajardo-Olivares, M., Rebollo-Vela, M., Vergara-Prieto, E., Blanco-Palenciano, J. (2007). Absceso preesternal por Salmonella enterica serovar. enteritidis. Enferm Infecc Microbiol Clin 25, 222.

9. Greenhow, T.L., (2019). 1526. Epidemiology of and Risk Factors for Nontyphoidal Salmonella Bacteremia in Children: A 20-Year Retrospective Cohort. Open Forum Infect Dis 6, S556.

10. Ao, T.T., Feasey, N.A., Gordon, M.A., Keddy, K.H., Angulo, F.J., Crump, J.A. (2015). Global Burden of Invasive Nontyphoidal Salmonella Disease, 20101. Emerg Infect Dis 21, 941-949. 
Ready to submit your research? Choose Auctores and benefit from:

* fast, convenient online submission

* rigorous peer review by experienced research in your field

* rapid publication on acceptance

* authors retain copyrights

* unique DOI for all articles

* immediate, unrestricted online access

At Auctores, research is always in progress.

Learn more www.auctoresonline.org/journals/clinical-medical-reviewsand-reports 